ORIGINAL ARTICLE

\title{
A Randomized Double-Blind Crossover Trial of Intradetrusor In- jection of Botulinum A Toxin for Severe and Refractory Idiopathic Detrusor Overactivity in Women
}

\author{
Paul Duggan* \\ Discipline of Obstetrics and Gynaecology, University of Adelaide and Gynaecology Unit, Central Adelaide \\ Local Health Network, Adelaide, Australia
}

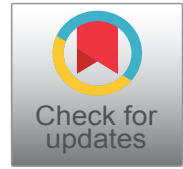

\begin{abstract}
*Corresponding author: Associate Professor Paul Duggan, MD FRANZCOG, Discipline of Obstetrics and Gynaecology, University of Adelaide and Gynaecology Unit, Central Adelaide Local Health Network, Adelaide, Australia, E-mail: paul.duggan@adelaide.edu.au
\end{abstract}

\begin{abstract}
Intradetrusor injection of onabotulinum A toxin (BTX-A) is an effective regimen for the highly prevalent condition in women of overactive bladder. The optimal dose of BTX-A has not been definitively established. Women $(n=13)$ with overactive bladder including urge incontinence refractory to at least two different oral/transdermal anticholinergic regimens and who had idiopathic detrusor overactivity were randomized to 100-unit and 200-unit doses utilizing a double-blinded crossover design. One woman (100-unit dose) experienced prolonged urinary retention. For the remaining patients, quality of life parameters estimated using the overactive bladder questionnaire baseline showed no significant difference between either dose regimen at 3, 6, 9 and 12 months follow up. Urodynamic data were recorded at (pre-treatment) and at 3 months after each treatment. Mean (SD) volume at first leakage was $178(82) \mathrm{ml}$ and $327(115) \mathrm{ml}$ for 200 units and $226(131) \mathrm{ml}$ and $215(139) \mathrm{ml}$ for 100 units $(p<0.001)$. Significant differences $(p<0.05)$ between baseline and 3 months in favour of the 200-unit dose were also observed in bladder capacity; volume at first urgency sensation; volume at first detrusor contraction. Changes in voiding parameters between baseline and 3 months for maximal flow rate, maximal detrusor pressure, and post-void residual volume were not significantly different for the two doses. Mean (SD) maximal voided volumes recorded on the patients' bladder diaries were $178(82) \mathrm{ml}$ and $327(115) \mathrm{ml}$ for 200 units and 226 (131) $\mathrm{ml}$ and 215 (139) $\mathrm{ml}$ for 100 units $(p<0.001)$. The following bladder diary parameters were not significantly different: daytime frequency; nocturia; incontinence episodes. Thus, for the subset of women with idiopathic detrusor overactivity, although urodynamic parameters suggest 200 units to be superior, this did not translate to any clinically meaningful improvement over 100 units.
\end{abstract}

\section{Keywords}

Idiopathic detrusor overactivity, Botulinum A toxin, Double blind, Randomized, Female

\begin{abstract}
Abbreviations
BTX-A: Onabotulinum Toxin; FDA: Food and Drug Administration; OAB: Overactive Bladder; IDO: Idiopathic Detrusor Overactivity; ICS: International Continence Society; IUGA: International Urogynaecological Association; NDO: Neuropathic Detrusor Overactivity; QoL: Quality of Life
\end{abstract}

\section{Introduction}

Overactive Bladder (OAB) defined jointly by the International Continence Society and International Urogynaecological Association (ICS/IUGA) is the unexplained clinical syndrome encompassing the symptoms of urinary urgency, frequency and nocturia, with or without urge urinary incontinence [1]. OAB has an estimated prevalence of $12.8 \%-43.1 \%$ in adult women and $10.8 \%-27.2 \%$ in adult men [2]. The overrepresentation of women is presumably related to factors such as anatomical differences (e.g. short female urethra), childbirth-related injury, hormonal effects, and psychological effects in some women, e.g. those who have experienced or continue to experience violence and sexual abuse. The broad range of treatment options for OAB attest to the difficulty in managing this condition. Non-invasive treatments for $O A B$ include behavioural modification, e.g. attention to fluid intake, timed voiding, and weight loss; pelvic floor muscle (Kegel) exercises; and prescribed

Citation: Duggan P (2017) A Randomized Double-Blind Crossover Trial of Intradetrusor Injection of Botulinum A Toxin for Severe and Refractory Idiopathic Detrusor Overactivity in Women. Int Arch Urol Complic 3:035. doi.org/10.23937/2469-5742/1510035

Received: June 10, 2017: Accepted: December 06, 2017: Published: December 08, 2017

Copyright: (c) 2017 Duggan P. This is an open-access article distributed under the terms of the Creative Commons Attribution License, which permits unrestricted use, distribution, and reproduction in any medium, provided the original author and source are credited. 
pharmacological regimens such as anticholinergics, alpha adrenergic agonists, and intravaginal oestrogen. Treatments to improve mobility and availability and mapping of utilities such as public toilets might help to prevent "accidents" due to urge incontinence. People of both genders who can't control urge incontinence will resort to incontinence aids such as pads, which are expensive and often uncomfortable. The condition often affects normal daily activities and sleeps and is a source of shame, embarrassment and social withdrawal. Quality of Life ( $\mathrm{QoL}$ ) is adversely affected and the prevalence of concomitant depression is increased [3]. Unfortunately, the non-invasive treatments described are often ineffective or the response to treatment suboptimal. This has driven the development of invasive treatments including sacral neuromodulation and the intradetrusor injection of botulinum A toxin.

There have been several reports published since 2005 on the use of Onabotulinum A Toxin (BTX-A) by intradetrusor injection for patients with neurogenic or idiopathic detrusor overactivity [4-10]. These data confirm superiority of BTX-A over placebo but were not definitive regarding the optimal dose, which were typically in the range of $50-200$ units for IDO and 200 300 units for NDO.

Dose-ranging studies for BTX-A in management of $O A B$ and with 8 or more episodes of urge incontinence a week concluded that a dose of 100 units optimally balances efficacy and risk of retention defined by arbitrary post void residual volumes $[11,12]$. The duration of benefit is variable, though efficacy is maintained with repeat treatments [13-19].

BTX-A by intradetrusor injection is now an established regimen for the treatment of $O A B$, and was approved in the United States by the FDA in 2014 for this purpose. In the USA, national guidelines are to use a dose of 100 units for $O A B$ [19]. This contrasts with the National Institute of Clinical Excellence (UK) guidelines, which recommend 200 units [20]. Internationally, for $O A B$ the optimal dose of BTX-A is not yet established [21].

In this trial, it was sought to establish which of two doses of BTX-A was the preferred option in management of women with $O A B$ with concomitant urge incontinence and who had IDO (as defined by ICS/IUGA) confirmed by urodynamic testing. The appropriateness of a policy of advising clean intermittent self-catheterisation determined by arbitrary post-void residual volumes has recently been challenged [22]. We did not set an arbitrary limit to post-void residual volumes and treated on symptoms alone. Thus, we hypothesised that the 200unit dose might prove superior with extended efficacy as determined by the patients' QoL.

\section{Methods and Materials}

\section{Context}

This trial was undertaken in the Royal Adelaide Hospital (RAH) - a publically-funded tertiary referral adult gynaecology unit in Adelaide, Australia. These women had initially presented elsewhere for management of $O A B$ and were later referred to our unit following failure of first line regimens. BTX-A was not at the commencement of this trial approved in Australia for the intended use, hence, approval for "off label" use was obtained from the Australian Therapeutic Goods Administration (TGA). At our institution, the use of "off label" regimens also required ethics approval and formal evaluation for hospital funding of the regimen. TGA approval was obtained in 2007. Originally, we planned a simple, randomised, crosssectional design - i.e., one dose, either $100 \mathrm{U}$ or $200 \mathrm{U}$ for each recruited patient. At that time, there were no data available to make power calculations. Based on our clinical load and an estimated $50 \%$ recruitment of patients with IDO, we expected we would in 2 years have completed 40 observations. That would have allowed a preliminary analysis of sufficient sample size from which to estimate the treatment effect and determine if the study was completed, viable but to continue, or non-viable and to cease. Recruitment of women to the trial proved difficult, primarily as most were not willing to accept the risk of prolonged urinary retention requiring clean intermittent self-catheterisation. We obtained expert statistical opinion, which was that switching to the crossover design described below would allow a greater probability of reaching a satisfactory conclusion with a smaller number of patients. We planned to undertake preliminary analysis after 20 patients had completed the crossover protocol. This required new ethics approval and the earlier cross-sectional data we had were unable to be used. Recruitment to the crossover trial also was slow and the study eventually was abandoned with our data reported here.

\section{Methodology}

Women with $\mathrm{OAB}$ who had confirmed, Idiopathic Detrusor Overactivity (IDO), normal voiding, and who had previously not responded to at least two anticholinergic regimens, and who did not have urodynamic stress incontinence were eligible for entry to the trial.

Full exclusion criteria are shown in Table 1. Although the patients were to be selected only if they had IDO, we treat symptoms, so the primary endpoint was quality of life (QoL). QoL was measured using the validated overactive bladder Quality of Life questionnaire (OABq) [23]. The OABq has two components - the Quality of Life Index (Range 0 - 100, higher numbers indicating better quality of life) and the Symptom Severity Index (range $0-100$, higher numbers indicating greater severity of symptoms). Patients completed the OABq at baseline, 6 weeks, 3 months and thereafter 3-monthly up until 12 months or until "treatment failure". BTX-A loses its effect at variable intervals. Such loss of effect was defined as "treatment failure" - a subjective secondary endpoint. Other secondary endpoints were changes in bladder diary data and urodynamic data between pretest baseline and repeated measures after treatment. 
Table 1: Exclusion criteria for eligibility to enroll in the trial.

Coexisting, untreated urinary stress incontinence

Concomitant use of:

- Anticholinergic regimens (to cease two weeks prior to treatment and remain off for 6 weeks post treatment)

- Anticoagulant or antiplatelet regimens (to cease pre-treatment and resume day 1 post treatment)

- Diuretics

Dementia

Hydronephrosis, post-void residual volume $>100 \mathrm{ml}$, or another urinary tract anomaly

Neurogenic detrusor overactivity

Pre-existing voiding abnormalities defined in the urodynamic clinic as either of:

- Uroflowmetry parameters (mean and maximal flow rates) $<10$ centile for age and gender (Liverpool nomogram)

- Post-void residual volumes $>100 \mathrm{ml}$

Pregnancy

Surgery for incontinence, vaginal prolapse or hysterectomy within 6 months of recruitment

Stroke or other cerebrovascular accident

Urinary tract infection

Unstable Diabetes

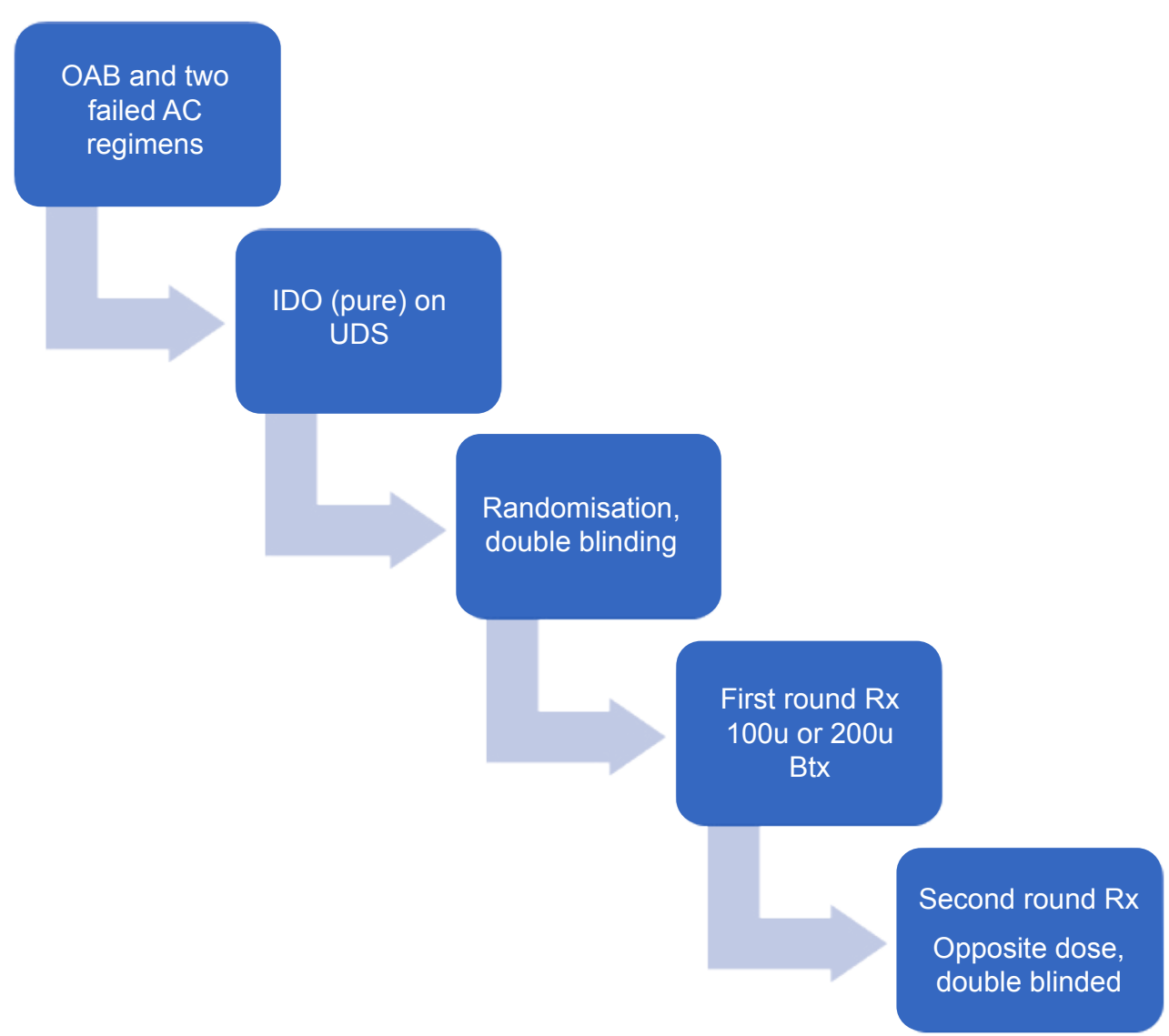

Figure 1: Flow diagram of the crossover design.

OAB: Overactive Bladder; AC: Anticholinergic; IDO: Idiopathic Detrusor Overactivity; UDS: Urodynamic Studies; Rx: Treatment; Btx: Onabotulinum A Toxin.

Patients were asked to record in their bladder diaries the times and amounts of fluid drunk and urine voided, and the time and nature of any abnormal urinary symptoms for 48 consecutive hours at the same time as recording $\mathrm{OABq}$ data. The 48-hour period of observation has in our experience been the most patient-friendly compromise and has achieved good compliance compared with longer intervals.

In this double-blind, randomized crossover trial (Figure 1), patients were centrally randomized in the hospital pharmacy to receive either 100 - or 200 -units at the first round of treatment and the opposite dose 
at the second (final) round of treatment. The timing of the second treatment was determined once the patient reported return of symptoms and was requesting retreatment. A repeat baseline evaluation including repeat urodynamic testing was arranged prior to the second intradetrusor injection of BTX-A. Women for whom treatment was successful have continued to receive treatment since the completion of the trial.

The BTX-A was diluted in $20 \mathrm{ml}$ normal saline by pharmacy staff not otherwise involved in the trial and presented in a pre-filled $20 \mathrm{ml}$ syringe in operating theatre labelled as BTX-A and with the patient's identifying information. The dilute BTX-A was administered by intradetrusor injection in $201 \mathrm{ml}$ aliquots in a $5 \times 4$ grid pattern with approximately equal spacing (avoiding visible subendothelial blood vessels) via a Williams 23 g cystoscopy needle and 30-degree, rigid cystoscope using a trigone sparing technique. Neither the patient, operating theatre staff, nor the researcher, had knowledge of the dose administered. The code held by pharmacy was only made available to the researcher once all follow up data had been collected.

Statistical analysis was undertaken using $\mathrm{IBM}^{\circledR} \mathrm{SPSS}^{\circledR}$ for Mac version 24. Analysis for paired OABq data was undertaken using the paired t-test and for other data by multivariate ANOVA. The duration of effect was estimated by computing the time taken following treatment for decay in the OABq Quality of Life Index to $20 \%$ above the baseline, or sooner if the patient requested to have the second treatment. These data were plotted using cumulative survival (Kaplan-Meier) analysis.

\section{Results}

Mean (SD) age at entry was 58 (13) years. One patient was indigenous Australian and the remainder white Caucasian. 12 of 13 women enrolled had both doses of BTX-A administered. One woman who received 100 units for the first treatment went in to prolonged urinary retention requiring catheterisation for 8 months and did not continue in the trial. Data were obtained from all remaining participants at baseline and 3-months post treatment. OABq data were available for 8 of the 12 patients at 9 months post-treatment, with the remainder having reported "treatment failure" before then. The full summary data for the Quality of Life and Severity Indices are shown in Table 2 and Table 3. For the Quality of Life Index, at 3 months post treatment, the mean (SD) difference was 1 (36) unit in favour of the 200-unit dose (this negligible difference was not statistically significant, $p=0.89$ ). At 9 months

Table 2: Baseline and post treatment Quality of Life Index (Overactive Bladder Questionnaire) for 100- and 200-unit doses of botulinum A toxin by intradetrusor injection for women with idiopathic detrusor overactivity.

\begin{tabular}{|l|l|l|l|l|l|}
\hline Quality of Life Index & Dose & Mean & Std. Error & \multicolumn{3}{|l|}{ 95\% Confidence Interval } \\
\hline Baseline & & & Lower Bound & Upper Bound \\
\hline Six Weeks & 100 & 48.6 & 13.3 & 11.8 & 85.5 \\
\cline { 2 - 6 } & 200 & 38.0 & 13.3 & 1.1 & 74.9 \\
\hline Three Months & 100 & 98.0 & 2.2 & 92.0 & 104.0 \\
\cline { 2 - 6 } & 200 & 96.3 & 2.2 & 90.3 & 102.4 \\
\hline Six Months & 100 & 97.0 & 2.7 & 89.4 & 104.5 \\
\hline Nine Months & 200 & 96.7 & 2.7 & 89.1 & 104.2 \\
\hline Twelve Months & 100 & 99.7 & 2.7 & 92.3 & 107.1 \\
\cline { 2 - 6 } & 200 & 94.3 & 2.7 & 86.9 & 101.7 \\
\hline & 100 & 97.3 & 4.2 & 85.7 & 109.0 \\
\hline
\end{tabular}

Table 3: Baseline and post treatment Severity Index (Overactive Bladder Questionnaire) for 100- and 200-unit doses of botulinum A toxin by intradetrusor injection for women with idiopathic detrusor overactivity.

\begin{tabular}{|l|l|l|l|l|l|}
\hline Severity Index & Dose & Mean & Std. Error & \multicolumn{2}{|l|}{ 95\% Confidence Interval } \\
\hline Baseline & & & & Lower Bound & Upper Bound \\
\hline Six Weeks & 100 & 62.3 & 9.7 & 35.4 & 89.2 \\
\hline & 200 & 64.7 & 9.7 & 37.8 & 91.5 \\
\hline Three Months & 100 & 2.3 & 2.2 & -3.7 & 8.4 \\
\hline Six Months & 200 & 3.3 & 2.2 & -2.7 & 9.4 \\
\hline Nine Months & 100 & 4.3 & 3.4 & -5.1 & 13.7 \\
\hline Twelve Months & 200 & 3.3 & 3.4 & -6.1 & 12.7 \\
\hline & 100 & 4.3 & 4.6 & -8.6 & 20.9 \\
\hline & 200 & 8.0 & 4.6 & -4.9 & 23.3 \\
\hline
\end{tabular}




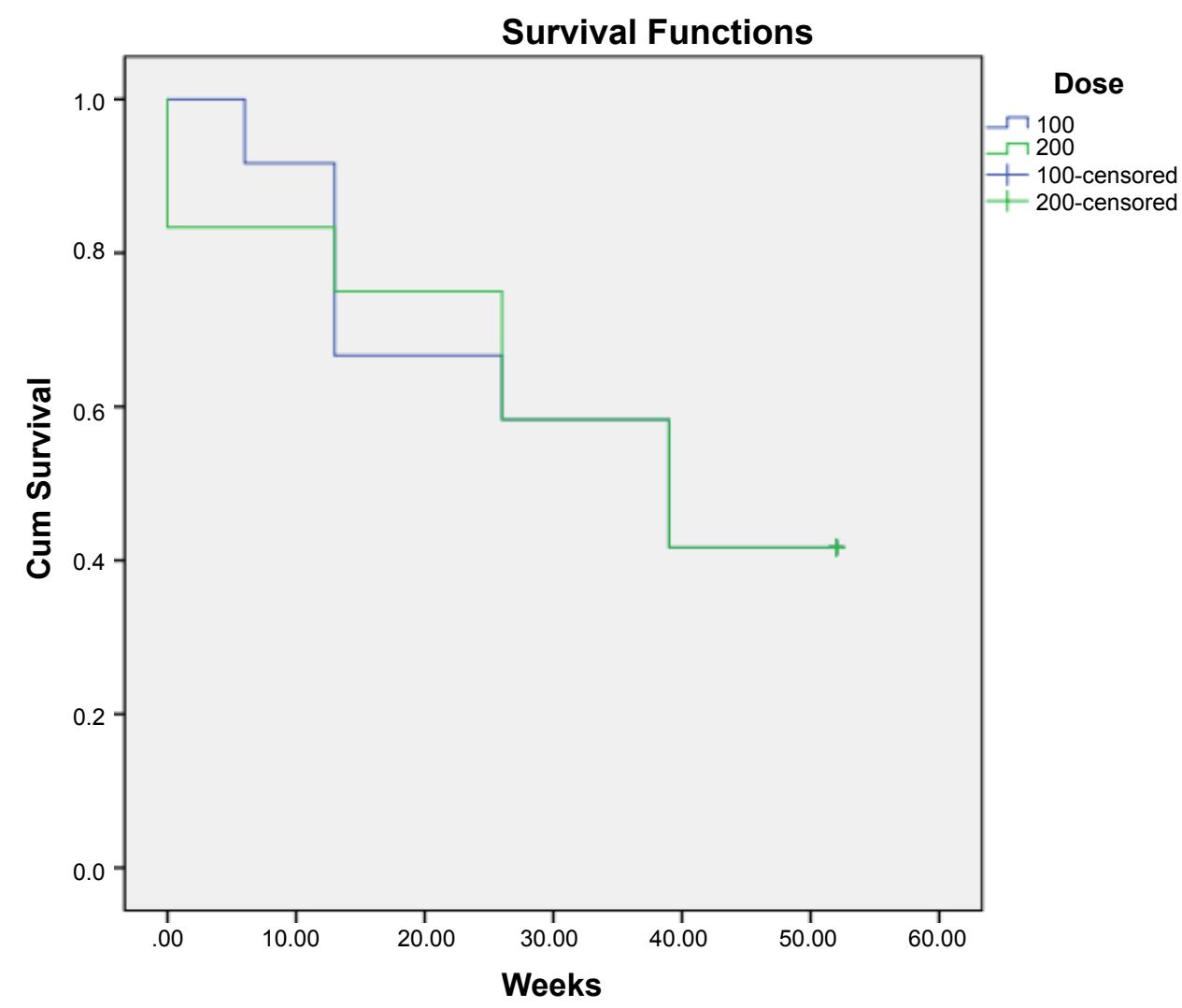

Figure 2: Duration of response to treatment (Cum Survival) determined by $20 \%$ or greater improvement over baseline of the Quality of Life index (Overactive Bladder Questionnaire) for 100- and 200-unit doses of botulinum a toxin by intradetrusor injection for women with idiopathic detrusor overactivity.

post treatment, there was no difference in quality of life with either dose. For the Severity Index, at 3 months post treatment, the mean (SD) difference was 10 (37) favouring the 100-unit dose (difference not significant, $p=0.63)$. At 9 months post treatment, this difference was 7 (33), also favouring the 100-unit dose (difference not significant, $p=0.56)$. Thus, for the primary endpoint 100 -units was equally effective as 200 units.

Survival analysis (Figure 2) calculated from the quality of life data shows a progressive decline in therapeutic effect over the 12 months of formal observation after each treatment. There was no difference in the rate of decline in effect between the two doses. 5 patients in both treatment groups reported persisting improvement at 12 months and did not require retreatment by the conclusion of this formal period of observation. 2 patients experienced this prolonged response with both doses.

Analysis based on the effect of the sequence of treatment (i.e. 100 units first or 200 units first) was undertaken and showed no effect, but the power of this observation was low.

Urodynamic data at baseline and 3 months post treatment are shown in Table 4. Volume at first leakage, cystometric bladder capacity, volume at first urgency sensation and volume at first detrusor contraction were all more favourable with the 200-unit dose regimen $(p<$ 0.05 ). Changes in voiding parameters between baseline and 3 months were not significantly different for the two doses.

Bladder diary parameters at baseline and at 3 months are shown in Table 5. The maximal voided volume increased to a significantly greater extent with the 200unit regimen $(p=0.04)$ but there was no difference in daytime frequency, nocturia or episodes of incontinence between dose regimens.

\section{Discussion}

This trial was established to determine the optimal dose of BTX-A given by intradetrusor injection in the subset of women presenting with refractory overactive bladder and who had an urodynamic diagnosis of idiopathic detrusor overactivity. It was assumed that either regimen would prove effective, or that BTX-A would in time be introduced as a standard option in clinical practice in our unit. As we would not have the funds or other capacity to offer a BTX-A regimen to every patient with $O A B$, we restricted eligibility to women with $O A B$ with urge incontinence refractory to two or more oral/transdermal anticholinergic regimens and who had IDO and in the absence of stress urinary incontinence, which is a confounder. We did not set an arbitrary limit to post-void residual volumes and treated on symptoms alone. We hypothesised that the 200unit dose might have superior efficacy as determined by the patients' QoL (the primary endpoint) given we were not arbitrarily recommending intermittent self- 
Table 4: Cystometry, uroflowmetry and pressure-flow study parameters at baseline and 3 months following treatment.

\begin{tabular}{|c|c|c|c|}
\hline Urodynamics Parameters - Cystometry & Baseline & Three Months & $p$ value \\
\hline \multicolumn{4}{|l|}{ Volume Fill } \\
\hline-100 units & $285(126)$ & $333(162)$ & \\
\hline - 200 units & $203(98)$ & $416(120)$ & 0.001 \\
\hline \multicolumn{4}{|l|}{ Volume First Sensation } \\
\hline-100 units & $117(68)$ & $170(110)$ & \\
\hline - 200 units & $108(53)$ & $238(116)$ & 0.06 \\
\hline \multicolumn{4}{|l|}{ Volume First Urgency Sensation } \\
\hline-100 units & $167(105)$ & $220(125)$ & \\
\hline - 200 units & $151(95)$ & $287(113)$ & 0.04 \\
\hline \multicolumn{4}{|l|}{ Volume First Detrusor Contraction } \\
\hline-100 units & $207(110)$ & $261(156)$ & \\
\hline - 200 units & $150(91)$ & $287(115)$ & $<0.05$ \\
\hline \multicolumn{4}{|l|}{ Volume First Leakage } \\
\hline-100 units & $226(131)$ & $215(139)$ & \\
\hline - 200 units & $178(82)$ & $327(115)$ & $<0.001$ \\
\hline \multicolumn{4}{|c|}{ Urodynamics Parameters -Uroflowmetry/Pressure Flow Studies } \\
\hline \multicolumn{4}{|c|}{ Volume Post Void Residual (averaged over two measures) } \\
\hline-100 units & $36(70)$ & $72(98)$ & \\
\hline - 200 units & $24(39)$ & $75(97)$ & 0.66 \\
\hline \multicolumn{4}{|l|}{ Maximal Flow Percentile } \\
\hline-100 units & $43(24)$ & $43(36)$ & \\
\hline - 200 units & $55(29)$ & $58(31)$ & 0.62 \\
\hline \multicolumn{4}{|l|}{ Maximal Detrusor Pressure } \\
\hline-100 units & $72(82)$ & $42(27)$ & \\
\hline - 200 units & $36(21)$ & $37(27)$ & 0.30 \\
\hline
\end{tabular}

Table 5: 48-hour bladder diary parameters at baseline and 3 months post treatment.

\begin{tabular}{|l|l|l|l|}
\hline Frequency Volume Chart Parameters & Baseline & Three Months & p value \\
\hline Frequency (awake) & & & \\
\hline-100 units & $11.0(4.3)$ & $8.7(2.9)$ & 0.84 \\
\hline-200 units & $9.9(3.8)$ & & \\
\hline & & & \\
\hline Nocturia & & $1.6(1.6)$ & 0.23 \\
\hline-100 units & $2.5(2.1)$ & $1.4(1.4)$ & \\
\hline-200 units & $2.7(1.4)$ & & \\
\hline Number of Times Incontinent & & & \\
\hline-100 units & $6.2(5.3)$ & $1.4(2.7)$ & 0.41 \\
\hline-200 units & $7.9(4.8)$ & $1.3(3.6)$ & \\
\hline Maximal Voided Volume & & & \\
\hline-100 units & & $340(147)$ & \\
\hline-200 units & $331(112)$ & $464(162)$ & \\
\hline
\end{tabular}

catheterisation in asymptomatic women.

The primary endpoint QoL was chosen because that is what patients hope to improve in undergoing such therapies and the $\mathrm{OABq}$ instrument chosen as it has been previously validated in the group of interest. The principal finding was that there was no difference in the primary endpoint whether a 100- or 200-unit dose was employed. This is counterintuitive, and the data bear examination to consider whether small numbers are responsible for this conclusion. i.e. a type I error. As the magnitude of the difference in the quality of life index was miniscule or absent (between 0 to 1 unit mean difference in a 100-unit range), it is very unlikely if larger numbers of patients had been studied that the conclusions would change. This can be corroborated by inspection of the survival analysis plot. By 12 months $38 \%$ of patients in either dose regimen was still happy with their bladder control - indicating natural selfselection for a favourable response in that subgroup, for reasons unexplained. This finding is consistent with observations by others [24].

A risk of intradetrusor BTX-A is prolonged post- 
treatment urinary retention, which significantly affected recruitment to this trial. Intuitively, this risk should be higher at the higher dose, and we might expect to see higher post void residuals in the 200-unit regimen. Unexpectedly, post void residual volumes at 3 months post treatment were indistinguishable, and this is unlikely to be a type I error. In this trial patients were required to have normal voiding parameters on entry, and, in contrast to previously reported studies, we did not plan to act on asymptomatic raised post void residual volumes. Unfortunately, one patient experienced severe urinary retention - able only to void very small volumes for approximately 8 months - and withdrew from the trial soon after her first treatment. This patient received the lower, 100-unit dose. Very prolonged urinary retention following intradetrusor injection of BTX-A has been reported previously [25]. It appears that patient response to BTX-A is idiosyncratic and, possibly, unpredictable. Using the lower 100-unit dose does not remove the risk of prolonged urinary retention.

Of the secondary endpoints, significant differences were observed in favour of the 200-unit dose for both sensory and motor cystometric parameters and also the patient record in the bladder diary of maximal voided volume. This, however, did not translate to any improvement in quality of life. A clue to why this might be so being in the bladder diary parameters: Frequency, nocturia and number of episodes of urinary incontinence which are indistinguishable between the two doses.

This study despite its small numbers has shown in our patient population that a 100-unit dose is the appropriate first option for treatment of women presenting with unexplained overactive bladder refractory to standard anticholinergic regimens and who have a urodynamic diagnosis of idiopathic detrusor overactivity. This result supports the current USA national guideline for treatment of overactive bladder, which is at variance with that of the UK $[19,20]$. This result, however, might not be generalisable to the population of women with overactive bladder and who have not had urodynamic testing. The women studied are representative of our local population, which in the age group studied is predominantly white Australian (Caucasian). However, the results are not necessarily generalisable to other populations. This research does not establish whether urodynamic testing offers any advantage in patient selection for BTX-A and has not addressed cost effectiveness in clinical practice.

\section{Acknowledgement}

This research was supported by a grant from the Australian Gynaecological Endoscopy Society. The support of the staff of the Royal Adelaide Hospital Pharmacy is gratefully acknowledged.

Ethical Statement (for Research Work Using Human or Animal Studies)
This study was approved by the Ethics Committee of the Royal Adelaide Hospital and the Ethics of Human Research Committee of the University of Adelaide.

\section{References}

1. Haylen BT, de Ridder D, Freeman RM, Swift SE, Berghmans B, et al. (2010). An international urogynecological association (IUGA)/international continence society (ICS) joint report on the terminology for female pelvic floor dysfunction. Neurourol Urodyn 29: 4-20.

2. Eapen R, Radomski SB (2016) Review of the epidemiology of overactive bladder. Res Rep Urol 8: 71-76.

3. Avery JC, Braunack-Mayer AJ, Duggan PM, Taylor AW, Stocks NP (2015) "It's our lot": how resilience influences the experience of depression in women with urinary incontinence". Health Sociology Review 24: 94-108.

4. Schurch B, de Seze M, Denys P, Chartier-Kastler E, Haab F, et al. (2005) Botox Detrusor Hyperreflexia Study Team. Botulinum toxin type $a$ is a safe and effective treatment for neurogenic urinary incontinence: results of a single treatment, randomized, placebo controlled 6-month study. J Urol 174: 196-200.

5. Werner M, Schmid DM, Schüssler B (2005) Efficacy of botulinum-A toxin in the treatment of detrusor overactivity incontinence: a prospective nonrandomized study. Am J Obstet Gynecol 192: 1735-1740.

6. Rajkumar GN, Small DR, Mustafa AW, Conn G (2005) A prospective study to evaluate the safety, tolerability, efficacy and durability of response of intravesical injection of botulinum toxin type $A$ into detrusor muscle in patients with refractory idiopathic detrusor overactivity. BJU Int 96: 848-852.

7. Popat R, Apostolidis A, Kalsi V, Gonzales G, Fowler CJ, et al. (2005) A comparison between the response of patients with idiopathic detrusor overactivity and neurogenic detrusor overactivity to the first intradetrusor injection of botulinum-A toxin. J Urol 174: 984-989.

8. Ghei M, Maraj BH, Miller R, Nathan S, O'Sullivan C, et al. (2005) Effects of botulinum toxin B on refractory detrusor overactivity: a randomized, double-blind, placebo controlled, crossover trial. J Urol 174: 1873-1877.

9. Tincello DG, Kenyon S, Abrams KR, Mayne C, Toozs-Hobson P, et al. (2012) Botulinum toxin a versus placebo for refractory detrusor overactivity in women: a randomised blinded placebo-controlled trial of 240 women (the RELAX study). Eur Urol 62: 507-514.

10. Sahai A, Dowson C, Khan MS, Dasgupta P (2010) Improvement in quality of life after botulinum toxin-A injections for idiopathic detrusor overactivity: results from a randomized double-blind placebo-controlled trial. BJU Int 103: 1509-1515.

11. Dmochowski R, Chapple C, Nitti VW, Chancellor M, Everaert K, et al. (2010) Efficacy and safety of onabotulinumtoxinA for idiopathic overactive bladder: a double-blind, placebo controlled, randomized, dose ranging trial. J Urol 184: 2416-2422.

12. Fowler CJ, Auerbach S, Ginsberg D, Hale D, Radziszewski $P$, et al. (2012) OnabotulinumtoxinA improves health-related quality of life in patients with urinary incontinence due to idiopathic overactive bladder: a 36-week, double-blind, placebo-controlled, randomized, dose-ranging trial. Eur Urol 62: 148-157.

13. Ginsberg DA, Drake MJ, Kaufmann A, Radomski S, Gousse AE, et al. (2017) Long-Term Treatment with OnabotulinumtoxinA Results in Consistent, Durable Improvements in 
Health-Related Quality of Life in Patients with Overactive Bladder. J Urol 198: 897-904.

14. Nitti VW, Dmochowski R, Herschorn S, Sand P, Thompson C, et al. (2017) OnabotulinumtoxinA for the Treatment of Patients with Overactive Bladder and Urinary Incontinence: Results of a Phase 3, Randomized, Placebo Controlled Trial. J Urol 197: S216-S223.

15. Nitti VW, Dmochowski R, Herschorn S, Sand P, Thompson C, et al. (2013) OnabotulinumtoxinA for the treatment of patients with overactive bladder and urinary incontinence: results of a phase 3 , randomized, placebo controlled trial. $J$ Urol 189: 2186-2193.

16. Chapple C, Sievert KD, MacDiarmid S, Khullar V, Radziszewski P, et al. (2013) OnabotulinumtoxinA $100 \mathrm{U}$ significantly improves all idiopathic overactive bladder symptoms and quality of life in patients with overactive bladder and urinary incontinence: a randomised, double-blind, placebo-controlled trial. Eur Urol 64: 249-256.

17. Denys $P$, Le Normand L, Ghout I, Costa P, Chartier-Kastler E, et al. (2012) VESITOX study group in France. Efficacy and safety of low doses of onabotulinumtoxinA for the treatment of refractory idiopathic overactive bladder: a multicentre, double-blind, randomised, placebo-controlled dose-ranging study. Eur Urol 61: 520-529.

18. Rovner E, Kennelly M, Schulte-Baukloh H, Zhou J, Haag-Molkenteller C, et al. (2011) Urodynamic results and clinical outcomes with intradetrusor injections of onabotulinumtoxinA in a randomized, placebo-controlled dose-finding study in idiopathic overactive bladder. Neurourol Urodyn 30: 556-562.
19. National Guideline Clearinghouse (NGC) (2014) Guideline summary: Diagnosis and treatment of overactive bladder (non-neurogenic) in adults: AUA/SUFU guideline. National Guideline Clearinghouse (NGC) Rockville (MD) Agency for Healthcare Research and Quality (AHRQ.

20. National Institute of Clinical Excellence (2013) Urinary Incontinence in Women: Management. Clinical Guideline CG171.

21. Nambiar A, Lucas M (2014) Chapter 4: Guidelines for the diagnosis and treatment of overactive bladder (OAB)and neurogenic detrusor overactivity (NDO). Neurourol Urodyn 33: S21-S25.

22. Collins L, Sathiananthamoorthy S, Fader M, Malone-Lee J (2017) Intermittent catheterisation after botulinum toxin injections: the time to reassess our practice. Int Urogynecol J 28: 1351-1356.

23. Coyne KS, Tubaro A, Brubaker L, Bavendam T (2006) Development and validation of patient-reported outcomes measures for overactive bladder: A review of concepts. Urology 68: 9-16.

24. Abdelwahab O, Sherif H, Soliman T, Elbarky I, Eshazly A (2015) Efficacy of botulinum toxin type A 100 Units versus 200 units for treatment of refractory idiopathic overactive bladder. Int Braz J Urol 41: 1132-1140.

25. King AB, Rapp DE (2011) Prolonged urinary retention after intravesical botulinum injection for treatment of idiopathic detrusor overactivity. Can J Urol 18: 5657-5659. 